\title{
Bifurcations of Orbit and Inclination Flips Heteroclinic Loop with Nonhyperbolic Equilibria
}

\author{
Fengjie Geng and Junfang Zhao \\ School of Science, China University of Geosciences (Beijing), Beijing, 100083, China \\ Correspondence should be addressed to Fengjie Geng; gengfengjie@cugb.edu.cn
}

Received 10 October 2013; Accepted 16 January 2014; Published 23 March 2014

Academic Editors: M. Han, Z. Jin, and Y. Xia

Copyright (c) 2014 F. Geng and J. Zhao. This is an open access article distributed under the Creative Commons Attribution License, which permits unrestricted use, distribution, and reproduction in any medium, provided the original work is properly cited.

\begin{abstract}
The bifurcations of heteroclinic loop with one nonhyperbolic equilibrium and one hyperbolic saddle are considered, where the nonhyperbolic equilibrium is supposed to undergo a transcritical bifurcation; moreover, the heteroclinic loop has an orbit flip and an inclination flip. When the nonhyperbolic equilibrium does not undergo a transcritical bifurcation, we establish the coexistence and noncoexistence of the periodic orbits and homoclinic orbits. While the nonhyperbolic equilibrium undergoes the transcritical bifurcation, we obtain the noncoexistence of the periodic orbits and homoclinic orbits and the existence of two or three heteroclinic orbits.
\end{abstract}

\section{Introduction}

In recent years, a great deal of mathematical efforts has been devoted to the bifurcation problems of homoclinic and heteroclinic orbits with high codimension, for example, the bifurcations of homoclinic or heteroclinic loop with orbit flip, the bifurcations of homoclinic or heteroclinic loop with inclination flip, and so forth; see [1-5] and the references therein. However, most of these papers considered the bifurcation problems of orbits connecting hyperbolic equilibria, and limited work has been done in the corresponding problems with nonhyperbolic equilibria; see [6-8]. To fill this gap, we investigate the bifurcations of orbit and inclination flip heteroclinic orbits with one nonhyperbolic equilibrium and one hyperbolic saddle. The method is using the fundamental solution matrix of the linear variational system to obtain the Poincaré map, which is easier to get the bifurcation equations.

Consider the following $C^{r}(r \geq 5)$ system

$$
\dot{z}=g(z, \lambda, \mu)
$$

and its unperturbed system

$$
\dot{z}=f(z),
$$

where $z \in \mathbb{R}^{4}$, the vector field $g$ depends on the parameters $(\lambda, \mu), \lambda \in \mathbb{R}, \mu \in \mathbb{R}^{l}, l \geq 2,0 \leq \lambda,|\mu| \ll 1, g(z, 0,0)=$ $f(z), g\left(p_{1}, 0, \mu\right)=0$, and $g\left(p_{2}, \lambda, \mu\right)=0$. Moreover, the parameter $\lambda$ governs bifurcation of the nonhyperbolic equilibrium, while $\mu$ controls bifurcations of the heteroclinic orbits.

Assuming system (2) has a heteroclinic loop $\Gamma$ connecting its two equilibria $p_{1}, p_{2}$, where $\Gamma=\Gamma^{1} \cup \Gamma^{2}, \Gamma^{i}=\left\{z=r_{i}(t)\right.$ : $t \in \mathbb{R}\}, r_{i}(+\infty)=r_{i+1}(-\infty)=p_{i+1}, i=1,2, r_{3}(t)=r_{1}(t)$, and $p_{3}=p_{1}$. Furthermore, the linearization $D f\left(p_{1}\right)$ has real eigenvalues $0, \lambda_{1}^{1},-\rho_{1}^{1}$, and $-\rho_{1}^{2}$ satisfying $-\rho_{1}^{2}<-\rho_{1}^{1}<0<$ $\lambda_{1}^{1} ; D f\left(p_{2}\right)$ has simple real eigenvalues $\lambda_{2}^{1}, \lambda_{2}^{2},-\rho_{2}^{1}$, and $-\rho_{2}^{2}$ fulfilling $-\rho_{2}^{2}<-\rho_{2}^{1}<0<\lambda_{2}^{1}<\lambda_{2}^{2}$.

The following conditions hold in the whole paper:

$$
e_{i}^{ \pm}=\lim _{t \rightarrow \pm \infty} \frac{\dot{r}_{i}(-t)}{\left|\dot{r}_{i}(-t)\right|},
$$

where $e_{1}^{+} \in T_{p_{1}} W_{1}^{c u}, e_{1}^{-} \in T_{p_{2}} W_{2}^{s s}, e_{2}^{+} \in T_{p_{2}} W_{2}^{u}, e_{2}^{-} \in T_{p_{1}} W_{1}^{s}$, and $e_{1}^{-} \in T_{p_{2}} W_{2}^{s s}$ mean that $\Gamma^{1}$ is a heteroclinic orbit with 
orbit flip, $W_{1}^{c u}$ is the center unstable manifold of $p_{1}, W_{i}^{u}$ (resp., $W_{i}^{s}$ ) is the unstable (resp., stable) manifold of $p_{i}$, and $W_{i}^{u u}$ (resp., $W_{i}^{s s}$ ) is the strong unstable (resp., stable) manifold of $p_{i}, i=1,2$. Moreover,

$$
\begin{aligned}
\operatorname{dim}( & \left.T_{r_{1}(t)} W_{1}^{\mathcal{c}} \cap T_{r_{1}(t)} W_{2}^{s}\right) \\
& =\operatorname{dim}\left(T_{r_{1}(t)} W_{1}^{\mathcal{c u}} \cap T_{r_{1}(t)} W_{2}^{s}\right)=1 .
\end{aligned}
$$

$\left(\mathrm{H}_{2}\right)$

$$
\begin{aligned}
& \lim _{t \rightarrow+\infty} T_{r_{1}(t)} W_{1}^{c u}=\operatorname{span}\left\{e_{1}^{-}, T_{p_{2}} W_{2}^{u u}\right\}, \\
& \lim _{t \rightarrow+\infty} T_{r_{2}(t)} W_{2}^{u}=\operatorname{span}\left\{e_{2}^{-}, T_{p_{1}} W_{1}^{u}\right\}, \\
& \lim _{t \rightarrow-\infty} T_{r_{1}(t)} W_{2}^{s}=\operatorname{span}\left\{e_{1}^{+}, T_{p_{1}} W_{1}^{s s}\right\}, \\
& \lim _{t \rightarrow-\infty} T_{r_{2}(t)} W_{1}^{s}=\operatorname{span}\left\{e_{2}^{+}, T_{p_{2}} W_{2}^{s}\right\},
\end{aligned}
$$

where the first three equations mean that the center unstable manifold $W_{1}^{c u}$ of $p_{1}$, the stable (resp., unstable) manifold $W_{2}^{s}$ (resp., $W_{2}^{u}$ ) of $p_{2}$ are fulfilling the strong inclination property. And the fourth equation implies that the stable manifold $W_{1}^{s}$ is of inclination flip as $t \rightarrow-\infty$.

It is worthy of noting that, for any integers $m \geq 1$ and $n \geq$ 1 , if we assume $\operatorname{dim}\left(W_{1}^{u}\right)=\operatorname{dim}\left(W_{2}^{u u}\right)=m$ and $\operatorname{dim}\left(W_{1}^{s s}\right)=$ $\operatorname{dim}\left(W_{2}^{s s}\right)=n$, then all the results achieved in this paper are still valid.

Let $\lambda \in \mathbb{R}$ be a parameter to control the transcritical bifurcation of system (1), let the $x$-axis be the tangent space of the center manifold at $p_{1}$, and let $\theta(x, \lambda, \mu)$ be the vector field defined on the center manifold; then by [9], we may assume

$$
\begin{aligned}
\left(H_{3}\right) & \theta\left(x_{p_{1}}, \lambda, \mu\right)=0,(\partial \theta / \partial x)\left(x_{p_{1}}, 0,0\right)=0, \\
& \left(\partial^{2} \theta / \partial x^{2}\right)\left(x_{p_{1}}, 0,0\right)>0,\left(\partial^{2} \theta / \partial x \partial\right)\left(x_{p_{1}}, 0,0\right)<0, \\
& \left(\partial^{2} \theta / \partial x \partial \mu\right)\left(x_{p_{1}}, 0, \mu\right)=0 \text {, where } x_{p_{1}} \text { is the } x \\
& \text { component of } p_{1} .
\end{aligned}
$$

If $\left(\mathrm{H}_{3}\right)$ is true, then system (1) exhibits the transcritical bifurcation, that is, when $\lambda>0$ (or $\lambda<0$; in this paper, we only consider the case $\lambda>0$; for the case $\lambda<0$, one may discuss it similarly); there are two hyperbolic saddles $p_{1}^{0}$ and $p_{1}^{1}$ bifurcated from $p_{1}$. Denote by $p_{1}^{0}=p_{1}=(0,0,0,0)^{*}$ and $p_{1}^{1}=p_{1}+\left(\lambda_{p}, 0,0,0\right)^{*}$, where $\lambda_{p}=\theta_{0} \lambda+O\left(\lambda^{2}\right)+O(\lambda \mu)$ and $\theta_{0}=-\left(\partial^{2} \theta / \partial x \partial \lambda\right)\left(x_{p_{1}}, 0,0\right) /\left(\partial^{2} \theta / \partial x^{2}\right)\left(x_{p_{1}}, 0,0\right)$. Moreover, $\operatorname{dim}\left(W_{p_{1}^{0}}^{s}\right)=3, \operatorname{dim}\left(W_{p_{1}^{0}}^{u}\right)=1$, and $\operatorname{dim}\left(W_{p_{1}^{1}}^{u}\right)=\operatorname{dim}\left(W_{p_{1}^{1}}^{s}\right)=$ 2 .

The present paper is built up as follows. In Section 2, we devote it to deriving the successor functions by constructing a suitable Poincaré Map. The analysis to the bifurcations of system (2) is presented in Section 3, where we establish the existence of the heteroclinic loop, the homoclinic orbits, and the three or two heteroclinic orbits and the coexistence of a periodic orbit and a homoclinic loop, and the difference between the heteroclinic loop with hyperbolic equilibria and nonhyperbolic equilibria is revealed.

\section{Normal Form and Poincaré Map}

Let the neighborhood $U_{i}$ of $p_{i}$ be small enough and straight the local manifolds of $W_{i}^{s}, W_{2}^{u u}, W_{i}^{s s}$, and $i=1,2$ in the neighborhood $U_{i}$. And then by virtue of the invariance of these manifolds and a scale transformation $x \rightarrow \theta_{x x}^{-1}\left(x_{p_{1}}, 0,0\right) x$ and $\lambda \rightarrow-\theta_{x \lambda}^{-1}\left(x_{p_{1}}, 0,0\right) \lambda$, system (1) has the following expression in $U_{1}$ :

$$
\begin{gathered}
\dot{x}=-\lambda_{p} x+x^{2}+O(u)[O(y)+O(v)] \\
+O(x)[O(y)+O(u)+O(v)]+O(x) O\left(x^{2}\right), \\
\dot{y}=\left[-\rho_{1}^{1}(\alpha)+\cdots\right] y+O(v)[O(x)+O(u)], \\
\dot{u}=\left[\lambda_{1}^{1}(\alpha)+\cdots\right] u+O(x)[O(y)+O(v)], \\
\dot{v}=\left[-\rho_{1}^{2}(\alpha)+\cdots\right] v+O(y)[O(x)+O(y)+O(u)],
\end{gathered}
$$

and in $U_{2}$ it takes the following form:

$$
\begin{gathered}
\dot{x}=\left[\lambda_{2}^{1}(\alpha)+\cdots\right] x+O(u)[O(y)+O(v)], \\
\dot{y}=\left[-\rho_{2}^{1}(\alpha)+\cdots\right] y+O(v)[O(x)+O(u)], \\
\dot{u}=\left[\lambda_{2}^{2}(\alpha)+\cdots\right] u+O(x)[O(x)+O(y)+O(v)], \\
\dot{v}=\left[-\rho_{2}^{2}(\alpha)+\cdots\right] v+O(y)[O(x)+O(y)+O(u)],
\end{gathered}
$$

where $\alpha=(\lambda, \mu), \lambda_{p}=\lambda+O\left(\lambda^{2}\right)+O(\lambda \mu), \lambda_{1}^{1}(0)=\lambda_{1}^{1}$, $\rho_{i}^{j}(0)=\rho_{i}^{j}, j=1,2, i=1,2, \lambda_{2}^{j}(0)=\lambda_{2}^{j}, j=1,2$.

From the normal form (6), (7), and the condition $\left(H_{1}\right)$, we may select $-T_{i}$ and $T_{i}$ such that

$$
\begin{array}{cc}
r_{1}\left(-T_{1}\right)=(\delta, 0,0,0)^{*}, & r_{1}\left(T_{1}\right)=(0,0,0, \delta)^{*}, \\
r_{1}\left(-T_{2}\right)=\left(\delta, 0,0, \delta_{u}, 0\right)^{*}, & r_{2}\left(T_{2}\right)=\left(0, \delta, 0, \delta_{v}\right)^{*},
\end{array}
$$

where $\delta>0$ is small enough such that $\{(x, y, u, v)$ : $|x|,|y|,|u|,|v|<2 \delta\} \subset U_{i}$ and $\left|\delta_{u}\right|=o(\delta),\left|\delta_{v}\right|=o(\delta)$.

Consider the linear variational system

$$
\dot{z}=D f\left(r_{i}(t)\right) z
$$

and its adjoint system

$$
\dot{\phi}=-\left(D f\left(r_{i}(t)\right)\right)^{*} \phi
$$

$i=1,2$, where $\left(D f\left(r_{i}(t)\right)\right)^{*}$ is the transposed matrix of $D f\left(r_{i}(t)\right)$.

Supposing $Z_{i}(t)=\left(z_{i}^{1}(t), z_{i}^{2}(t), z_{i}^{3}(t), z_{i}^{4}(t)\right)$ is a fundamental solution matrix of $(9)_{i}$, then, we arrive at the following lemma. 
Lemma 1. If conditions $\left(H_{1}\right)-\left(H_{3}\right)$ are satisfied, then

(1) there exists a fundamental solution matrix of (9) satisfying

$$
\begin{aligned}
& z_{1}^{1}(t) \in\left(T_{r_{1}(t)} W_{1}^{c u}\right)^{c} \cap\left(T_{r_{1}(t)} W_{2}^{s}\right)^{c}, \\
& z_{1}^{2}(t)=-\frac{\dot{r}_{1}(t)}{\left|\dot{r}_{1}\left(T_{1}\right)\right|} \in T_{r_{1}(t)} W_{1}^{c} \cap T_{r_{1}(t)} W_{2}^{s}, \\
& z_{1}^{3}(t) \in T_{r_{1}(t)} W_{1}^{c u} \cap\left(T_{r_{1}(t)} W_{2}^{s}\right)^{c}, \\
& z_{1}^{4}(t) \in\left(T_{r_{1}(t)} W_{1}^{c u}\right)^{c} \cap T_{r_{1}(t)} W_{2}^{s}
\end{aligned}
$$

such that

$$
\begin{gathered}
Z_{1}\left(-T_{1}\right)=\left(\begin{array}{cccc}
w_{1}^{11} & w_{1}^{21} & 0 & w_{1}^{41} \\
w_{1}^{12} & 0 & 0 & w_{1}^{42} \\
w_{1}^{13} & w_{1}^{23} & 1 & w_{1}^{43} \\
0 & 0 & 0 & w_{1}^{44}
\end{array}\right), \\
Z_{1}\left(T_{1}\right)=\left(\begin{array}{cccc}
1 & 0 & w_{1}^{31} & 0 \\
\widetilde{w}_{1}^{12} & 0 & w_{1}^{32} & 1 \\
0 & 0 & w_{1}^{33} & 0 \\
0 & 1 & w_{1}^{34} & 0
\end{array}\right)
\end{gathered}
$$

(2) $(9)_{2}$ has a fundamental solution matrix fulfilling

$$
\begin{aligned}
& z_{2}^{1}(t) \in\left(T_{r_{2}(t)} W_{2}^{u}\right)^{c} \cap\left(T_{r_{2}(t)} W_{1}^{s}\right)^{c}, \\
& z_{2}^{2}(t)=-\frac{\dot{r}_{2}(t)}{\left|\dot{r}_{2}\left(T_{2}\right)\right|} \in T_{r_{2}(t)} W_{2}^{u} \cap T_{r_{2}(t)} W_{1}^{s}, \\
& z_{2}^{3}(t) \in T_{r_{2}(t)} W_{2}^{u} \cap\left(T_{r_{2}(t)} W_{1}^{s}\right)^{c}, \\
& z_{2}^{4}(t) \in\left(T_{r_{2}(t)} W_{2}^{u}\right)^{c} \cap T_{r_{2}(t)} W_{1}^{s}
\end{aligned}
$$

such that

$$
\begin{aligned}
& Z_{2}\left(-T_{2}\right)=\left(\begin{array}{cccc}
w_{2}^{11} & w_{2}^{21} & 0 & w_{2}^{41} \\
0 & 0 & 0 & w_{2}^{42} \\
w_{2}^{13} & w_{2}^{23} & 1 & w_{2}^{43} \\
w_{2}^{14} & 0 & 0 & 0
\end{array}\right), \\
& Z_{2}\left(T_{2}\right)=\left(\begin{array}{cccc}
1 & 0 & w_{2}^{31} & 0 \\
0 & 1 & w_{2}^{32} & 0 \\
0 & 0 & w_{2}^{33} & 0 \\
\widetilde{w}_{2}^{14} & w_{2}^{24} & w_{2}^{34} & 1
\end{array}\right),
\end{aligned}
$$

where $w_{i}^{21}<0, w_{1}^{12} w_{i}^{33} w_{2}^{14} w_{2}^{42} \neq 0,\left|\left(w_{i}^{33}\right)^{-1} w_{i}^{3 j}\right| \ll 1, j=$ $1,2,4, i=1,2$.

Now, let $\left(z_{i}^{1}(t), z_{i}^{2}(t), z_{i}^{3}(t), z_{i}^{4}(t)\right)$ be a new local active coordinate system along $\Gamma^{i}$. Given $\Phi_{i}(t)=$ $\left(\phi_{i}^{1}(t), \phi_{i}^{2}(t), \phi_{i}^{3}(t), \phi_{i}^{4}(t)\right)=\left(Z_{i}^{-1}(t)\right)^{*}$, then $\Phi_{i}(t)$ is the fundamental solution matrix of $(10)_{i}, i=1,2$.

Let $z=r_{i}(t)+Z_{i}(t) N_{i}(t) \triangleq h_{i}(t)$, where $N_{i}(t)=$ $\left(n_{i}^{1}, 0, n_{i}^{3}, n_{i}^{4}\right)^{*}, i=1,2$. Defining the cross sections

$$
\begin{aligned}
& S_{i}^{0}=\left\{z=h_{i}\left(-T_{i}\right):|x|,|y|,|u|,|v|<2 \delta\right\}, \\
& S_{i}^{1}=\left\{z=h_{i}\left(T_{i}\right):|x|,|y|,|u|,|v|<2 \delta\right\}
\end{aligned}
$$

of $\Gamma_{i}$ at $t=-T_{i}$ and $t=T_{i}$, respectively, $i=1,2$.
Now that if $q_{i}^{0} \in S_{i}^{0}$ and $q_{i}^{1} \in S_{i}^{1}$, then

$$
\begin{gathered}
q_{i}^{0}=\left(x_{i}^{0}, y_{i}^{0}, u_{i}^{0}, v_{i}^{0}\right)^{*}=r_{i}\left(-T_{i}\right)+Z_{1}\left(-T_{i}\right) N_{i}\left(-T_{i}\right), \\
N_{i}\left(-T_{i}\right)=\left(n_{i}^{0,1}, 0, n_{i}^{0,3}, n_{i}^{0,4}\right)^{*}, \\
q_{i}^{1}=\left(x_{i}^{1}, y_{i}^{1}, u_{i}^{1}, v_{i}^{1}\right)^{*}=r_{i}\left(T_{i}\right)+Z_{i}\left(T_{i}\right) N_{i}\left(T_{i}\right), \\
N_{i}\left(T_{i}\right)=\left(n_{i}^{1,1}, 0, n_{i}^{1,3}, n_{i}^{1,4}\right)^{*} .
\end{gathered}
$$

Based on the expressions of $Z_{i}\left(-T_{i}\right)$ and $Z_{i}\left(T_{i}\right)$, we get their new coordinates of $q_{i}^{0}\left(n_{i}^{0,1}, 0, n_{i}^{0,3}, n_{i}^{0,4}\right)^{*}$ and $q_{i}^{1}\left(n_{i}^{1,1}, 0, n_{i}^{1,3}, n_{i}^{1,4}\right)^{*}$; that is,

$$
\begin{aligned}
n_{1}^{0,1}= & \left(w_{1}^{12}\right)^{-1}\left[y_{1}^{0}-w_{1}^{42}\left(w_{1}^{44}\right)^{-1} v_{1}^{0}\right], \\
n_{1}^{0,3}= & u_{1}^{0}-w_{1}^{13}\left(w_{1}^{12}\right)^{-1} y_{1}^{0} \\
& +\left[w_{1}^{13} w_{1}^{42}\left(w_{1}^{12}\right)^{-1}-w_{1}^{43}\right]\left(w_{1}^{44}\right)^{-1} v_{1}^{0}, \\
n_{1}^{0,4}= & \left(w_{1}^{44}\right)^{-1} v_{1}^{0}, \\
x_{1}^{0}= & \delta+w_{1}^{11} n_{1}^{0,1}+w_{1}^{41} n_{1}^{0,4} \approx \delta, \\
n_{1}^{1,1}= & x_{1}^{1}-w_{1}^{31}\left(w_{1}^{33}\right)^{-1} u_{1}^{1}, \\
n_{1}^{1,3}= & \left(w_{1}^{33}\right)^{-1} u_{1}^{1}, \\
n_{1}^{1,4}= & y_{1}^{1}-\widetilde{w}_{1}^{12} x_{1}^{1}+\left(\widetilde{w}_{1}^{12} w_{1}^{31}-w_{1}^{32}\right)\left(w_{1}^{33}\right)^{-1} u_{1}^{1}, \\
v_{1}^{1} \approx & \delta \\
n_{2}^{0,1}= & \left(w_{2}^{14}\right)^{-1} v_{2}^{0}, \\
n_{2}^{0,3}= & u_{2}^{0}-\delta_{2}^{u}-w_{2}^{13}\left(w_{2}^{14}\right)^{-1} v_{2}^{0}-w_{2}^{43}\left(w_{2}^{42}\right)^{-1} y_{2}^{0}, \\
n_{2}^{0,4}= & \left(w_{2}^{42}\right)^{-1} y_{2}^{0}, \\
x_{2}^{0} \approx & \delta \\
n_{2}^{1,1}= & x_{0}^{1}-w_{2}^{31}\left(w_{2}^{33}\right)^{-1} u_{0}^{1}, \\
n_{2}^{1,3}= & \left(w_{2}^{33}\right)^{-1} u_{0}^{1}, \\
n_{2}^{1,4}= & v_{0}^{1}-\delta_{2}^{v}-\widetilde{w}_{2}^{14} x_{0}^{1}+\left(\widetilde{w}_{2}^{14} w_{2}^{31}-w_{2}^{34}\right)\left(w_{2}^{33}\right)^{-1} u_{0}^{1}, \\
y_{0}^{1} \approx & \delta \\
& \\
&
\end{aligned}
$$

Next, we divide our establishment of the Poincaré map in the new coordinate system in three steps. 
First, consider the map $F_{i}^{1}: S_{i}^{0} \mapsto S_{i}^{1}$. Put $z=h_{i}(t)$ into (1); we have

$$
\begin{aligned}
\dot{r}_{i}(t) & +\dot{Z}_{i}(t) N_{i}(t)+Z_{i}(t) \dot{N}_{i}(t) \\
= & g\left(r_{i}(t)+Z_{i}(t) N_{i}(t), \lambda, \mu\right) \\
= & g\left(r_{i}(t), 0,0\right)+g_{z}\left(r_{i}(t), 0,0\right) Z_{i}(t) N_{i}(t) \\
& +g_{\lambda}\left(r_{i}(t), 0,0\right) \lambda+g_{\mu}\left(r_{i}(t), 0,0\right) \mu+\text { h.o.t. } \\
= & f\left(r_{i}(t)\right)+D f\left(r_{i}(t)\right) Z_{i}(t) N_{i}(t) \\
& +g_{\lambda}\left(r_{i}(t), 0,0\right) \lambda+g_{\mu}\left(r_{i}(t), 0,0\right) \mu+\text { h.o.t. }
\end{aligned}
$$

According to the fact $\dot{r}_{i}(t)=f\left(r_{i}(t)\right)$ and $\dot{Z}_{i}(t)=$ $D f\left(r_{i}(t)\right) Z_{i}(t)$, it then yields to that

$$
\begin{aligned}
\dot{N}_{i}(t)= & Z_{i}^{-1}(t)\left[g_{\lambda}\left(r_{i}(t), 0,0\right) \lambda+g_{\mu}\left(r_{i}(t), 0,0\right) \mu\right] \\
& + \text { h.o.t. }
\end{aligned}
$$

Integrating the above equation from $-T_{i}$ to $T_{i}$, we arrive at

$$
\begin{aligned}
N_{i}\left(T_{i}\right)= & N_{i}\left(-T_{i}\right)+\int_{-T_{i}}^{T_{i}} Z_{i}^{-1}(t) g_{\lambda}\left(r_{i}(t), 0,0\right) \lambda d t \\
& +\int_{-T_{i}}^{T_{i}} Z_{i}^{-1}(t) g_{\mu}\left(r_{i}(t), 0,0\right) \mu d t+\text { h.o.t. }
\end{aligned}
$$

Noticing that $\Phi_{i}^{*}(t)=Z_{i}^{-1}(t)$, then

$$
n_{i}^{1, j}=n_{i}^{0, j}+M_{i \lambda}^{j} \lambda+M_{i \mu}^{j} \mu+\text { h.o.t., } \quad j=1,3,4,
$$

where

$$
\begin{gathered}
M_{i \lambda}^{j}=\int_{-T_{i}}^{T_{i}} \phi_{i}^{j *} g_{\lambda}\left(r_{i}(t), 0,0\right) d t \\
M_{i \mu}^{j}=\int_{-T_{i}}^{T_{i}} \phi_{i}^{j *} g_{\mu}\left(r_{i}(t), 0,0\right) d t, \quad j=1,3,4 .
\end{gathered}
$$

Together with (17) and $(21)_{i},(22)_{i}$ then defines the map $F_{i}^{1}$ : $S_{i}^{0} \mapsto S_{i}^{1},\left(n_{i}^{0,1}, 0, n_{i}^{0,3}, n_{i}^{0,4}\right) \mapsto\left(n_{i}^{1,1}, 0, n_{i}^{1,3}, n_{i}^{1,4}\right)$.

Next, to construct the map $F_{i}^{0}: S_{i-1}^{1} \mapsto S_{i}^{0}$ (where $S_{0}^{1}=S_{2}^{1}$ ). Let $\tau_{i}, i=1,2$ be the flying time from $q_{i-1}^{1}\left(x_{i-1}^{1}, y_{i-1}^{1}, u_{i-1}^{1}, v_{i-1}^{1}\right)^{*}$ to $q_{i}^{0}\left(x_{i}^{0}, y_{i}^{0}, u_{i}^{0}, v_{i}^{0}\right)^{*}$; set $s_{1}=$ $e^{-\rho_{1}^{1}(\alpha) \tau_{1}}$ and $s_{2}=e^{-\rho_{2}^{1}(\alpha) \tau_{2}}$. By virtue of the approximate solution of system (6) and (7), if we neglect the higher terms, then the expression of $F_{1}^{0}: S_{0}^{1} \mapsto S_{1}^{0}$ is

$$
\begin{gathered}
x_{0}^{1} \approx \frac{x_{1}^{0}}{h\left(s_{1}\right)}, \quad y_{1}^{0} \approx s_{1} y_{0}^{1}, \\
u_{0}^{1} \approx s_{1}^{\lambda_{1}^{1}(\alpha) / \rho_{1}^{1}(\alpha)} u_{1}^{0}, \quad v_{1}^{0} \approx s_{1}^{\rho_{1}^{2}(\alpha) / \rho_{1}^{1}(\alpha)} v_{0}^{1}
\end{gathered}
$$

and $F_{2}^{0}: S_{1}^{1} \mapsto S_{2}^{1}$ is

$$
\begin{gathered}
x_{1}^{1} \approx s_{2}^{\lambda_{2}^{1}(\alpha) / \rho_{2}^{1}(\alpha)} x_{2}^{0}, \quad y_{2}^{0} \approx s_{2} y_{1}^{1}, \\
u_{1}^{1} \approx s_{2}^{\lambda_{2}^{2}(\alpha) / \rho_{2}^{1}(\alpha)} u_{2}^{0}, \quad v_{2}^{0} \approx s_{2}^{\rho_{2}^{2}(\alpha) / \rho_{2}^{1}(\alpha)} v_{1}^{1},
\end{gathered}
$$

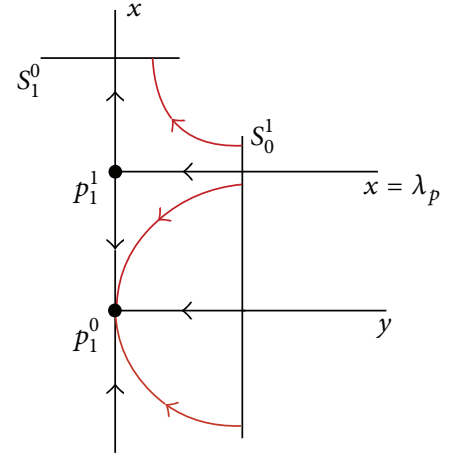

Figure 1

where $\left(s_{i}, u_{i}^{0}, v_{i-1}^{1}\right), i=1,2$ are called Shilnikov coordinates, and

$$
h(s)= \begin{cases}\left(\lambda_{p}\right)^{-1}\left[x_{1}^{0}-\left(x_{1}^{0}-\lambda_{p}\right) s^{\lambda_{p} / \rho_{1}^{1}(\alpha)}\right], & \lambda_{p} \neq 0, \\ 1-\left(\rho_{1}^{1}(\alpha)\right)^{-1} x_{1}^{0} \ln s, & \lambda_{p}=0 .\end{cases}
$$

Since the nonhyperbolic equilibrium $p_{1}$ undergoes a transcritical bifurcation based on the structure of orbits in $U_{1}$, we may see that the equation $x_{0}^{1} \approx x_{1}^{0} / h\left(s_{1}\right)$ holds only for $x_{0}^{1} \geq \lambda_{p}$. While for $x_{0}^{1} \in\left[-\beta, \lambda_{p}\right)(0<\beta \ll 1)$, the map $F_{1}^{0}$ is well defined only if $s_{1}=0$ (see Figure 1). So, we extend the domain of $F_{1}^{0}$, defining

$$
x_{1}^{0}=\delta, \quad s_{1}=0, \quad \text { if } x_{0}^{1} \in\left[-\beta, \lambda_{p}\right) .
$$

The final step is to compose the maps $F_{i}^{0}$ and $F_{i}^{1}$, and then $F_{1}=F_{1}^{1} \circ F_{1}^{0}: S_{0}^{1} \mapsto S_{1}^{1}$ can be expressed as

$$
\begin{aligned}
n_{1}^{1,1}= & \left(w_{1}^{12}\right)^{-1} \delta s_{1}-\left(w_{1}^{12}\right)^{-1} w_{1}^{42}\left(w_{1}^{44}\right)^{-1} s_{1}^{\rho_{1}^{2}(\alpha) / \rho_{1}^{1}(\alpha)} v_{0}^{1} \\
& +M_{1 \lambda}^{1} \lambda+M_{1 \mu}^{1} \mu+\text { h.o.t., } \\
n_{1}^{1,3}= & u_{1}^{0}-w_{1}^{13}\left(w_{1}^{12}\right)^{-1} \delta s_{1} \\
& +\left[w_{1}^{13} w_{1}^{42}\left(w_{1}^{12}\right)^{-1}-w_{1}^{43}\right]\left(w_{1}^{44}\right)^{-1} s_{1}^{\rho_{1}^{2}(\alpha) / \rho_{1}^{1}(\alpha)} v_{0}^{1} \\
& +M_{1 \lambda}^{3} \lambda+M_{1 \mu}^{3} \mu+\text { h.o.t., } \\
n_{1}^{1,4}= & \left(w_{1}^{44}\right)^{-1} s_{1}^{\rho_{1}^{2}(\alpha) / \rho_{1}^{1}(\alpha)} v_{0}^{1}+M_{1 \lambda}^{4} \lambda+M_{1 \mu}^{4} \mu+\text { h.o.t. }
\end{aligned}
$$

and $F_{2}=F_{2}^{1} \circ F_{2}^{0}: S_{1}^{1} \mapsto S_{2}^{1}\left(=S_{0}^{1}\right)$ as

$$
\begin{aligned}
n_{2}^{1,1}= & \left(w_{2}^{14}\right)^{-1} \delta s_{2}^{\rho_{2}^{2}(\alpha) / \rho_{2}^{1}(\alpha)}+M_{2 \lambda}^{1} \lambda+M_{2 \mu}^{1} \mu+\text { h.o.t., } \\
n_{2}^{1,3}= & u_{2}^{0}-\delta_{2}^{u}-w_{2}^{13}\left(w_{2}^{14}\right)^{-1} \delta s_{2}^{\rho_{2}^{2}(\alpha) / \rho_{2}^{1}(\alpha)} \\
& -w_{2}^{43}\left(w_{2}^{42}\right)^{-1} s_{2} y_{1}^{1}+M_{2 \lambda}^{3} \lambda+M_{2 \mu}^{3} \mu+\text { h.o.t., } \\
n_{2}^{1,4}= & \left(w_{2}^{44}\right)^{-1} s_{2} y_{1}^{1}+M_{2 \lambda}^{4} \lambda+M_{2 \mu}^{4} \mu+\text { h.o.t. }
\end{aligned}
$$


Set $G_{i}=F_{i}\left(q_{i-1}^{1}\right)-q_{i}^{1}, i=1,2$. Combing $(21)_{i},(23),(24)$, (27), and (28), we derive the successor functions $G_{i}^{j}$ :

$$
\begin{aligned}
G_{1}^{1}= & \left(w_{1}^{12}\right)^{-1} \delta s_{1}-\delta s_{2}^{\lambda_{2}^{1}(\alpha) / \rho_{2}^{1}(\alpha)}+M_{1 \lambda}^{1} \lambda+M_{1 \mu}^{1} \mu+\text { h.o.t., } \\
G_{1}^{3}= & u_{1}^{0}-w_{1}^{13}\left(w_{1}^{12}\right)^{-1} \delta s_{1}-\left(w_{1}^{33}\right)^{-1} s_{2}^{\lambda_{2}^{2}(\alpha) / \rho_{2}^{1}(\alpha)} u_{2}^{0} \\
& +M_{1 \lambda}^{3} \lambda+M_{1 \mu}^{3} \mu+\text { h.o.t., } \\
G_{1}^{4}= & \left(w_{1}^{44}\right)^{-1} s_{1}^{\rho_{1}^{2}(\alpha) / \rho_{1}^{1}(\alpha)} v_{0}^{1}-y_{1}^{1}+\widetilde{w}_{1}^{12} \delta s_{2}^{\lambda_{2}^{1}(\alpha) / \rho_{2}^{1}(\alpha)} \\
& +M_{1 \lambda}^{4} \lambda+M_{1 \mu}^{4} \mu+\text { h.o.t., } \\
G_{2}^{1}= & \left(w_{2}^{14}\right)^{-1} \delta s_{2}^{\rho_{2}^{2}(\alpha) / \rho_{2}^{1}(\alpha)}-\delta h\left(s_{1}\right) \\
& +w_{2}^{31}\left(w_{2}^{33}\right)^{-1} s_{1}^{\lambda_{1}^{1}(\alpha) / \rho_{1}^{1}(\alpha)} u_{1}^{0}+M_{2 \lambda}^{1} \lambda+M_{2 \mu}^{1} \mu+\text { h.o.t. } \\
G_{2}^{3}= & u_{2}^{0}-\delta_{2}^{u}-w_{2}^{13}\left(w_{1}^{14}\right)^{-1} \delta s_{2}^{\rho_{2}^{2}(\alpha) / \rho_{2}^{1}(\alpha)} \\
& -w_{2}^{43}\left(w_{2}^{42}\right)^{-1} s_{2}^{\rho_{2}^{1}(\alpha) / \rho_{2}^{1}(\alpha)} y_{1}^{1} \\
& -\left(w_{2}^{33}\right)^{-1} s_{1}^{\lambda_{1}^{1}(\alpha) / \rho_{1}^{1}(\alpha)} u_{1}^{0}+M_{2 \lambda}^{3} \lambda+M_{2 \mu}^{3} \mu+\text { h.o.t., } \\
G_{2}^{4}= & \left(w_{2}^{44}\right)^{-1} s_{2} y_{1}^{1}-v_{0}^{1}+\delta_{2}^{v}+\widetilde{w}_{2}^{14} \delta h\left(s_{1}\right) \\
& -\left(w_{2}^{34}-\widetilde{w}_{2}^{14} w_{2}^{31}\right)\left(w_{2}^{33}\right)^{-1} s_{1}^{\lambda_{1}^{1}(\alpha) / \rho_{1}^{1}(\alpha)} u_{1}^{0} \\
& +M_{2 \lambda}^{4} \lambda+M_{2 \mu}^{4} \mu+\text { h.o.t. }
\end{aligned}
$$

It is easy to see that what we need to do is considering the solutions of

$$
\left(G_{1}^{1}, G_{1}^{3}, G_{1}^{4}, G_{2}^{1}, G_{2}^{3}, G_{2}^{4}\right)=0
$$

with $s_{1} \geq 0$ and $s_{2} \geq 0$. This is because the solution of (30) with $s_{1}=s_{2}=0$ (resp., $s_{1}>0, s_{2}>0 ; s_{1}=0, s_{2}>0$ or $s_{1}>0$, $s_{2}=0$ ) means that system (1) has a heteroclinic loop (resp., a periodic orbit; homoclinic loop).

\section{Main Results}

Based on the expressions of the successor functions and the implicit function theorem, we know that the equation $\left(G_{1}^{3}, G_{1}^{4}, G_{2}^{3}, G_{2}^{4}\right)=0$ has a unique solution $\left(u_{1}^{0}, u_{2}^{0}, y_{1}^{1}, v_{0}^{1}\right)$. And putting it into $\left(G_{1}^{1}, G_{2}^{1}\right)=0$, then we obtain the following bifurcation equations:

$$
\begin{aligned}
&\left(w_{1}^{12}\right)^{-1} \delta s_{1}-\delta s_{2}^{\lambda_{2}^{1} / \rho_{2}^{1}}+M_{1 \lambda}^{1} \lambda+M_{1 \mu}^{1} \mu+\text { h.o.t. }=0, \\
&\left(w_{2}^{14}\right)^{-1} \delta s_{2}^{\rho_{2}^{2} / \rho_{2}^{1}}-\delta h\left(s_{1}\right)+M_{2 \lambda}^{1} \lambda+M_{2 \mu}^{1} \mu \\
&+w_{2}^{31}\left(w_{2}^{33}\right)^{-1} s_{1}^{\lambda_{1}^{1} / \rho_{1}^{1}}\left[w_{1}^{13}\left(w_{1}^{12}\right)^{-1} \delta s_{1}+\left(w_{1}^{33}\right)^{-1}\right. \\
& \times s_{2}^{\lambda_{2}^{2} / \rho_{2}^{1}}\left(\delta_{2}^{u}-M_{2 \lambda}^{3} \lambda-M_{2 \mu}^{3} \mu\right) \\
&\left.-M_{1 \lambda}^{3} \lambda-M_{1 \mu}^{3} \mu\right]+ \text { h.o.t. }=0 .
\end{aligned}
$$

Firstly, we consider the case $\lambda=0$, which means the transcritical bifurcation does not happen. By (23) and (25), (31) turns to

$$
\begin{aligned}
& \left(w_{1}^{12}\right)^{-1} \delta s_{1}-\delta s_{2}^{\lambda_{2}^{1} / \rho_{2}^{1}}+M_{1 \mu}^{1} \mu+\text { h.o.t. }=0, \\
& \left(w_{2}^{12}\right)^{-1} \delta s_{2}^{\rho_{2}^{2} / \rho_{2}^{1}}-\frac{\delta}{1-\left(\rho_{1}^{1}\right)^{-1} \delta \ln s_{1}}+M_{2 \mu}^{1} \mu \\
& +w_{2}^{31}\left(w_{2}^{33}\right)^{-1} w_{1}^{13}\left(w_{1}^{12}\right)^{-1} \delta s_{1}^{\lambda_{1}^{1} / \rho_{1}^{1}+1} \\
& -w_{2}^{31}\left(w_{2}^{33}\right)^{-1} s_{1}^{\lambda_{1}^{1} / \rho_{1}^{1}} M_{1 \mu}^{3} \mu+\text { h.o.t. }=0 .
\end{aligned}
$$

Noticing that $\lambda_{1}^{1} / \rho_{1}^{1}>0$, which shows $\lim _{s_{1} \rightarrow 0} s_{1}^{\lambda_{1}^{1} / \rho_{1}^{1}}(1-$ $\left.\left(\rho_{1}^{1}\right)^{-1} \delta \ln s_{1}\right)=0$, it then follows that

$$
\begin{gathered}
s_{1}-w_{1}^{12} s_{2}^{\lambda_{2}^{1} / \rho_{2}^{1}}+\delta^{-1} w_{1}^{12} M_{1 \mu}^{1} \mu+\text { h.o.t. }=0 \\
\left(w_{2}^{12}\right)^{-1} s_{2}^{\rho_{2}^{2} / \rho_{2}^{1}}-\frac{1}{1-\left(\rho_{1}^{1}\right)^{-1} \delta \ln s_{1}}+\delta^{-1} M_{2 \mu}^{1} \mu+\text { h.o.t. }=0 .
\end{gathered}
$$

From the above bifurcation equations, we obtain the following results immediately.

Theorem 2. Let the conditions $\left(H_{1}\right)-\left(H_{3}\right)$ be true and $M_{i \mu}^{1} \neq 0$, $i=1,2$. Then, for $\lambda=0$ and $0<|\mu| \ll 1$, one has

(i) for $\operatorname{rank}\left(M_{1 \mu}^{1}, M_{2 \mu}^{1}\right)=2$, there exists a codimension 2 surface

$$
L_{12}=\left\{\mu: M_{1 \mu}^{1} \mu+\text { h.o.t. }=M_{2 \mu}^{1} \mu+\text { h.o.t. }=0\right\}
$$

such that system (1) has a unique heteroclinic loop near $\Gamma$ if and only if $\mu \in L_{12}$, where the surface $L_{12}$ has a normal plane $\operatorname{span}\left\{M_{1 \mu}^{1}, M_{2 \mu}^{1}\right\}$ at $\mu=0$.

(ii) there exists an $(l-1)$-dimensional surface

$$
\begin{gathered}
L_{1}^{2}=\left\{\mu: \delta^{-1} w_{2}^{12} M_{2 \mu}^{1} \mu+\left(\delta^{-1} M_{1 \mu}^{1} \mu\right)^{\rho_{2}^{2} / \lambda_{2}^{1}}+\text { h.o.t. }=0,\right. \\
\left.M_{1 \mu}^{1} \mu>0\right\} \\
\left(\text { resp., } L_{2}^{1}=\left\{\mu: \frac{\delta}{1-\left(\rho_{1}^{1}\right)^{-1} \delta \ln \left(-\delta^{-1} w_{1}^{12} M_{1 \mu}^{1} \mu\right)}\right.\right. \\
\left.\left.-M_{2 \mu}^{1} \mu+\text { h.o.t. }=0, w_{1}^{12} M_{1 \mu}^{1} \mu<0\right\}\right)
\end{gathered}
$$

such that system (1) has a unique homoclinic loop connecting $p_{1}$ (resp., connecting $p_{2}$ ) near $\Gamma$ if and only if $\mu \in L_{1}^{2}$ (resp., $\left.\mu \in L_{2}^{1}\right)$. 
Proof. The result (i) will be proved by putting $s_{1}=s_{2}=0$ into (33).

If we assume $s_{1}=0$ and $s_{2}>0$ in (33), then

$$
\begin{gathered}
s_{2}^{\lambda_{2}^{1} / \rho_{2}^{1}}=\delta^{-1} M_{1 \mu}^{1} \mu+\text { h.o.t., } \\
\left(w_{2}^{12}\right)^{-1} s_{2}^{\rho_{2}^{2} / \rho_{2}^{1}}+\delta^{-1} M_{2 \mu}^{1} \mu+\text { h.o.t. }=0,
\end{gathered}
$$

which means

$$
\left(w_{2}^{12}\right)^{-1} s_{2}^{\rho_{2}^{2} / \lambda_{2}^{1}}+\delta^{-1} M_{2 \mu}^{1} \mu+\text { h.o.t. }=0 .
$$

It follows that there exists an $(l-1)$-dimensional surface $L_{1}^{2}$ given by (35) such that (33) has a unique solution $s_{1}=0$, $s_{2}=s_{2}(\mu)>0$ as $\mu \in L_{1}^{2}$ and $0<|\mu| \ll 1$. This implies system (1) has a homoclinic loop connecting $p_{1}$. The existence of $L_{2}^{1}$ can be obtained similarly.

This completes the proof.

Remark 3. There is no difficulty to see that $L_{1}^{2}$ has a normal vector $M_{2 \mu}^{1}$ at $\mu=0$ as $\rho_{2}^{2}>\lambda_{2}^{1}$, while for $\rho_{2}^{2}<\lambda_{2}^{1}$ (resp., $\rho_{2}^{2}>\lambda_{2}^{1}$ ) it has a normal vector $M_{1 \mu}^{1}$ (resp., $\left.M_{1 \mu}^{1}+w_{2}^{12} M_{2 \mu}^{1}\right)$ at $\mu=0$.

Theorem 4. Assume the conditions $\left(H_{1}\right)-\left(H_{3}\right)$ hold and $M_{i \mu}^{1} \neq 0, i=1,2$. Then for $\lambda=0, \mu \in L_{1}^{2}$, and $0<|\mu| \ll 1$, the periodic orbit and homoclinic loop with $p_{1}$ of system (1) cannot coexist.

Proof. Theorem 2 shows that if $\mu \in L_{1}^{2}$ and $0<|\mu| \ll 1$, then system (1) has a homoclinic loop with $p_{1}$. Setting $s_{1} \geq 0$, $s_{2}^{\lambda_{2}^{1} / \rho_{2}^{1}}=\left(w_{1}^{12}\right)^{-1} s_{1}+\delta^{-1} M_{1 \mu}^{1} \mu+$ h.o.t. $>0$, and $\mu \in L_{1}^{2}$, then (33) is reduced to

$$
\begin{aligned}
V_{1}\left(s_{1}\right) \triangleq & {\left[\left(w_{1}^{12}\right)^{-1} s_{1}+\delta^{-1} M_{1 \mu}^{1} \mu\right]^{\rho_{2}^{2} / \lambda_{2}^{1}}+\delta^{-1} w_{2}^{12} M_{2 \mu}^{1} \mu } \\
& + \text { h.o.t. }=\frac{w_{2}^{12}}{1-\left(\rho_{1}^{1}\right)^{-1} \delta \ln s_{1}} \triangleq N_{1}\left(s_{1}\right) .
\end{aligned}
$$

Notice that $V_{1}(0)=N_{1}(0)$ and

$$
\begin{gathered}
V_{1}^{\prime}\left(s_{1}\right)=\frac{\rho_{2}^{2}}{\lambda_{2}^{1}}\left(w_{1}^{12}\right)^{-1}\left[\left(w_{1}^{12}\right)^{-1} s_{1}+\delta^{-1} M_{1 \mu}^{1} \mu\right]^{\rho_{2}^{2} / \lambda_{2}^{1}-1} \\
N_{1}^{\prime}\left(s_{1}\right)=\frac{w_{2}^{12}\left(\rho_{1}^{1}\right)^{-1} \delta}{\left(1-\left(\rho_{1}^{1}\right)^{-1} \delta \ln s_{1}\right)^{2} s_{1}}
\end{gathered}
$$

If $w_{1}^{12} w_{2}^{12}<0$, then $V_{1}^{\prime}\left(s_{1}\right) N_{1}^{\prime}\left(s_{1}\right)<0$; it is obvious that $V_{1}\left(s_{1}\right)=N_{1}\left(s_{1}\right)$ has no sufficiently small positive solutions.

While $\rho_{2}^{2}>\lambda_{2}^{1}$, then $\left|V_{1}^{\prime}\left(s_{1}\right)\right| \ll 1$ and $\left|N_{1}^{\prime}\left(s_{1}\right)\right| \gg 1$ hold for $0<s_{1} \ll 1$, which shows that $V_{1}\left(s_{1}\right)=N_{1}\left(s_{1}\right)$ has no sufficiently small positive solution.

Next, we only consider the case $\rho_{2}^{2} \leq \lambda_{2}^{1}$ and $w_{1}^{12} w_{2}^{12}>0$. As $\mu \in L_{1}^{2}$, we have $M_{1 \mu}^{1} \mu>0$, and then, for $w_{i}^{12}>0, i=1,2$ we see that

$$
V_{1}^{\prime}\left(s_{1}\right) \leq\left(w_{1}^{12}\right)^{-\rho_{2}^{2} / \lambda_{2}^{1}} s_{1}^{\rho_{2}^{2} / \lambda_{2}^{1}-1}<N_{1}^{\prime}\left(s_{1}\right) \quad \text { for } 0<s_{1} \ll 1 .
$$

In fact, $\rho_{2}^{2}<\lambda_{2}^{1}$ yields that $\lim _{s_{1} \rightarrow 0^{+}} s_{1}^{\rho_{2}^{2} / \lambda_{2}^{1}-1}=+\infty$, $\lim _{s_{1} \rightarrow 0^{+}} N_{1}^{\prime}\left(s_{1}\right)=+\infty$, and $\lim _{s_{1} \rightarrow 0} s_{1}^{\rho_{2}^{2} / \lambda_{2}^{1}-1} / N_{1}^{\prime}\left(s_{1}\right)=0$, which shows $V_{1}\left(s_{1}\right)=N_{1}\left(s_{1}\right)$ has no sufficiently small positive solutions. Obviously, the conclusion is correct as $\rho_{2}^{2}=\lambda_{2}^{1}$.

Similarly, for $\rho_{2}^{2}<\lambda_{2}^{1}, w_{i}^{12}<0, i=1,2$, there does not exist a small positive solution for $V_{1}\left(s_{1}\right)=N_{1}\left(s_{1}\right)$.

The proof is then completed.

Theorem 5. Assume that the conditions $\left(H_{1}\right)-\left(H_{3}\right)$ hold and $M_{i \mu}^{1} \neq 0, i=1,2$. Let $\lambda=0, \mu \in L_{2}^{1}$, and $0<|\mu| \ll 1$; then

(i) the periodic orbit and the homoclinic loop connecting $p_{2}$ of system (1) cannot coexist as $\rho_{2}^{2} \geq \lambda_{2}^{1}$ or $w_{1}^{12} w_{2}^{12}<0$;

(ii) at least one periodic orbit and the homoclinic loop connecting $p_{2}$ of system (1) coexist as $\rho_{2}^{2}<\lambda_{2}^{1}, w_{1}^{12}>0$, and $w_{2}^{12}>0$;

(iii) a unique periodic orbit and the homoclinic loop connecting $p_{2}$ of system (1) coexist as $\rho_{2}^{2}<\lambda_{2}^{1}, w_{1}^{12}<0$, and $w_{2}^{12}<0$.

Proof. By Theorem 2, the condition $\mu \in L_{2}^{1}$ for $0<|\mu| \ll 1$ implies that system (1) has a homoclinic loop connecting $p_{2}$.

(i) Let $s_{2}=e^{-\rho_{2}^{1} \tau_{2}}$ and eliminating $s_{1}$ in (33), we derive

$$
\begin{aligned}
V_{2}\left(s_{2}\right) & \triangleq s_{2}+\delta^{-1} w_{2}^{12} M_{2 \mu}^{1} \mu+\text { h.o.t. } \\
& =\frac{w_{2}^{12}}{1-\left(\rho_{1}^{1}\right)^{-1} \delta \ln \left(w_{1}^{12}\left(s_{2}^{\lambda_{2}^{1} / \rho_{2}^{2}}-\delta^{-1} M_{1 \mu}^{1} \mu\right)\right)} \\
& \triangleq N_{2}\left(s_{2}\right) .
\end{aligned}
$$

Note that $V_{2}(0)=N_{2}(0)$ as $\mu \in L_{2}^{1}$. Moreover,

$$
\begin{gathered}
V_{2}^{\prime}\left(s_{2}\right)=1 \\
N_{2}^{\prime}\left(s_{2}\right)=\frac{w_{2}^{12}\left(\rho_{1}^{1}\right)^{-1} \delta}{\left[1-\left(\rho_{1}^{1}\right)^{-1} \delta \ln \left(w_{1}^{12}\left(s_{2}^{\lambda_{2}^{1} / \rho_{2}^{2}}-\delta^{-1} M_{1 \mu}^{1} \mu\right)\right)\right]^{2}} \\
\cdot \frac{\left(\lambda_{2}^{1} / \rho_{2}^{2}\right) s_{2}^{\left(\lambda_{2}^{1}-\rho_{2}^{2}\right) / \rho_{2}^{2}}}{\left(s_{2}^{\lambda_{2}^{1} / \rho_{2}^{2}}-\delta^{-1} M_{1 \mu}^{1} \mu\right)} .
\end{gathered}
$$

For $\rho_{2}^{2} \geq \lambda_{2}^{1}$ and $N_{2}^{\prime}\left(s_{2}\right) \ll 1=V_{2}^{\prime}\left(s_{2}\right)$, this means $V_{2}\left(s_{2}\right)=$ $\mathrm{N}_{2}\left(s_{2}\right)$ has no sufficiently small positive solutions.

Now we turn to the case $w_{1}^{12} w_{2}^{12}<0$, since we are interested in sufficiently small positive solutions of (33), it suffices to consider the sufficiently small positive solutions of $V_{2}\left(s_{2}\right)=N_{2}\left(s_{2}\right)$ satisfying $w_{1}^{12}\left(s_{2}^{\lambda_{2}^{1} / \rho_{2}^{2}}-\delta^{-1} M_{1 \mu}^{1} \mu\right)>0$, which implies that $s_{2}^{\lambda_{2}^{1} / \rho_{2}^{2}}-\delta^{-1} M_{1 \mu}^{1} \mu<0$ (resp., $s_{2}^{\lambda_{2}^{1} / \rho_{2}^{2}}-\delta^{-1} M_{1 \mu}^{1} \mu>$ 0 ) for $w_{1}^{12}<0$ (resp., $w_{1}^{12}>0$ ). It is easy to see that $V_{2}\left(s_{2}\right)=N_{2}\left(s_{2}\right)$ has no sufficiently small positive solutions as $w_{1}^{12} w_{2}^{12}<0$. 
(ii) For $\rho_{2}^{2}<\lambda_{2}^{1}$, we have $V_{2}^{\prime}(0)=1>0=N_{2}^{\prime}(0)$, which implies that there exists an $0<\widetilde{s}_{2} \ll 1$ such that $V_{2}\left(s_{2}\right)>$ $N_{2}\left(s_{2}\right)$ for $0<s_{2}<\widetilde{s}_{2}$.

Choosing $\widehat{s}_{2}=\delta^{-1} w_{2}^{12} M_{2 \mu}^{1} \mu>0$, then

$$
\begin{gathered}
V_{2}\left(\widehat{s}_{2}\right)=2 \delta^{-1} w_{2}^{12} M_{2 \mu}^{1} \mu+\text { h.o.t., } \\
N_{2}\left(\widehat{s}_{2}\right)=\frac{w_{2}^{12}}{1-\left(\rho_{1}^{1}\right)^{-1} \delta \ln \left(w_{1}^{12}\left(\hat{s}_{2}^{\lambda_{2}^{1} / \rho_{2}^{2}}-\delta^{-1} M_{1 \mu}^{1} \mu\right)\right)} .
\end{gathered}
$$

In view of $\ln \left(w_{1}^{12}\left(\widehat{s}_{2}^{\lambda_{2}^{1} / \rho_{2}^{2}}-\delta^{-1} M_{1 \mu}^{1} \mu\right)\right)>\ln \left(w_{1}^{12} \widehat{s}_{2}^{1}{ }_{2}^{1} / \rho_{2}^{2}\right)=$ $\ln \left(w_{1}^{12}\left(\delta^{-1} w_{2}^{12} M_{2 \mu}^{1} \mu\right)^{\lambda_{2}^{1} / \rho_{2}^{2}}\right)$ for $w_{1}^{12}>0$, so

$$
\begin{aligned}
N_{2}\left(\widehat{s}_{2}\right)> & \frac{w_{2}^{12}}{1-\left(\rho_{1}^{1}\right)^{-1} \delta \ln \left(w_{1}^{12}\left(w_{1}^{12}\left(\delta^{-1} w_{2}^{12} M_{2 \mu}^{1} \mu\right)^{\lambda_{2}^{1} / \rho_{2}^{2}}\right)\right)} \\
& \gg 2 w_{1}^{12}\left(\delta^{-1} w_{2}^{12} M_{2 \mu}^{1} \mu\right)=V_{2}\left(\widehat{s}_{2}\right)
\end{aligned}
$$

when $w_{2}^{12}>0$. As a result, $N_{2}\left(s_{2}\right)=V_{2}\left(s_{2}\right)$ has at least one solution $\bar{s}_{2}$ satisfying $0<\widetilde{s}_{2}<\bar{s}_{2}<\widehat{s}_{2} \ll 1$.

(iii) $s_{2}$ must fulfill $0<s_{2}<\left(\delta^{-1} M_{1 \mu}^{1} \mu\right)^{\rho_{2}^{2} / \lambda_{2}^{1}}$ as $w_{1}^{12}<0$; with similar arguments in proof of (ii), we can prove that there exists a $0<s_{2}^{*} \ll 1$ such that $V_{2}\left(s_{2}^{*}\right)=N_{2}\left(s_{2}^{*}\right)$ for $0<s_{2}^{*}<$ $\left(\delta^{-1} M_{1 \mu}^{1} \mu\right)^{\rho_{2} / \lambda_{2}^{1}} \ll 1$. It is easy to compute that $N_{2}^{\prime \prime}\left(s_{2}\right)>0$ for $w_{2}^{12}<0,0<s_{2}<\left(\delta^{-1} M_{1 \mu}^{1} \mu\right)^{\rho_{2}^{2} / \lambda_{2}^{1}}$, and $\mu \in L_{2}^{1}$. Combining with the fact $V_{2}(0)=N_{2}(0), N_{2}^{\prime}\left(s_{2}\right)>0$, and $V_{2}^{\prime}\left(s_{2}\right)=1$, we immediately know that $s_{2}^{*}$ is unique.

This completes the proof.

Now, we turn to discussing the bifurcations of the heteroclinic loop for $\lambda>0$, when $p_{1}$ undergoes a transcritical bifurcation. From Figure 1, we know that when $\lambda>0$, after the creation of the equilibria $p_{1}^{0}$ and $p_{1}^{1}$, there always exists a straight segment orbit heteroclinic to $p_{1}^{1}$ and $p_{1}^{0}$, its length is $\lambda_{p}$, and we denote this heteroclinic orbit by $\Gamma^{*}$. Moreover, $x_{0}^{1}=\lambda_{p}$ is a critical position.

(31) becomes

Firstly, we take into account the case $x_{0}^{1} \geq \lambda_{p}$. In this case,

$$
\begin{gathered}
\left(w_{1}^{12}\right)^{-1} \delta s_{1}-\delta s_{2}^{\lambda_{2}^{1} / \rho_{2}^{1}}+M_{1 \lambda}^{1} \lambda+M_{1 \mu}^{1} \mu+\text { h.o.t. }=0, \\
\left(w_{2}^{12}\right)^{-1} \delta s_{2}^{\rho_{2}^{2} / \rho_{2}^{1}}-\delta \lambda_{p}\left[\delta-\left(\delta-\lambda_{p}\right) s_{1}^{\lambda_{p} / \rho_{1}^{1}}\right]^{-1} \\
+M_{2 \lambda}^{1} \lambda+M_{2 \mu}^{1} \mu \\
+w_{2}^{31}\left(w_{2}^{33}\right)^{-1} s_{1}^{\lambda_{1}^{1} / \rho_{1}^{1}}\left[w_{1}^{13}\left(w_{1}^{12}\right)^{-1} \delta s_{1}+\left(w_{1}^{33}\right)^{-1} \delta s_{2}^{\lambda_{2}^{2} / \lambda_{2}^{1}}\right. \\
\left.-M_{1 \lambda}^{3} \lambda-M_{1 \mu}^{3} \mu\right]+ \text { h.o.t. }=0 .
\end{gathered}
$$

Let $s=s_{1}^{\lambda_{p} / \rho_{1}^{1}}\left(s=0\right.$ means $s_{1}=0$ and vice versa); by virtue of Taylor's development for $\delta \lambda_{p} /\left(\delta-\left(\delta-\lambda_{p}\right) s_{1}{ }^{\lambda_{p} / \rho_{1}^{1}}\right)$, we have

$$
\begin{gathered}
\left(w_{1}^{12}\right)^{-1} s^{\rho_{1}^{1} / \lambda_{p}}-s_{2}^{\lambda_{2}^{1} / \rho_{2}^{1}}+\delta^{-1} M_{1 \lambda}^{1} \lambda+\delta^{-1} M_{1 \mu}^{1} \mu+\text { h.o.t. }=0 \\
\left(w_{2}^{12}\right)^{-1} \delta s_{2}^{\rho_{2}^{2} / \rho_{2}^{1}}-\lambda_{p}-\frac{\lambda_{p}\left(\delta-\lambda_{p}\right)}{\delta} s+M_{2 \lambda}^{1} \lambda \\
+M_{2 \mu}^{1} \mu+\text { h.o.t. }=0 .
\end{gathered}
$$

With similar arguments to $\lambda=0$, we may easily obtain the following results.

Theorem 6. Suppose the conditions $\left(H_{1}\right)-\left(H_{3}\right)$ hold, $0<\lambda \ll$ 1 ; then

(i) if $\operatorname{rank}\left(M_{1 \mu}^{1}, M_{2 \mu}^{1}\right)=2$, there exists an $(l-2)$ dimensional surface

$$
\begin{aligned}
L_{12}^{\lambda}=\{\mu(\lambda): & M_{1 \mu}^{1} \mu+M_{1 \lambda}^{1} \lambda+h . o . t . \\
& \left.=M_{2 \mu}^{1} \mu+M_{2 \lambda}^{1} \lambda-\lambda+\text { h.o.t. }=0\right\}
\end{aligned}
$$

such that system (1) has a unique heteroclinic loop if and only if $\mu \in L_{12}^{\lambda}$ and $0<|\mu| \ll 1$;

(ii) there exists an $(l-1)$-dimensional surface

$$
\begin{aligned}
& L_{1 \lambda}^{2}=\left\{\mu(\lambda): W_{1}^{2}(\lambda, \mu)=\right.\left(w_{2}^{12}\right)^{-1}\left[\delta^{-1}\left(M_{1 \mu}^{1} \mu+M_{1 \lambda}^{1} \lambda\right)\right]^{\beta_{2}} \\
&+\delta^{-1}\left(M_{2 \mu}^{1} \mu+M_{2 \lambda}^{1} \lambda\right) \\
&-\delta^{-1} \lambda_{p}+\text { h.o.t. }=0, \\
&\left.M_{1 \mu}^{1} \mu+M_{1 \lambda}^{1} \lambda>0\right\}
\end{aligned}
$$

(resp.,

$$
\begin{aligned}
& L_{2 \lambda}^{1}=\left\{\mu(\lambda): W_{2}^{1}(\lambda, \mu)\right. \\
&= \delta \lambda_{p}+\lambda_{p}\left(\delta-\lambda_{p}\right) \\
& \times\left[-\delta^{-1} w_{1}^{12}\left(M_{1 \lambda}^{1} \lambda+M_{1 \mu}^{1} \mu\right)\right]^{\lambda_{p} / \rho_{1}^{1}} \\
&-\delta M_{2 \lambda}^{1} \lambda \\
&-\delta M_{2 \mu}^{1} \mu+\text { h.o.t. }=0, \\
&\left.\left.w_{1}^{12}\left(M_{1 \lambda}^{1} \lambda+M_{1 \mu}^{1} \mu\right)<0\right\}\right)
\end{aligned}
$$

such that system (1) has one homoclinic loop connecting $p_{1}^{1}$ (resp., connecting $p_{2}$ ) if and only if $\mu \in L_{1 \lambda}^{2}$ and $0<|\mu| \ll 1$.

Theorem 7. Suppose hypotheses $\left(H_{1}\right)-\left(H_{3}\right)$ hold, $M_{i \mu}^{1} \neq 0, i=$ $1,2,0<\lambda,|\mu| \ll 1$, and $w_{1}^{12} w_{2}^{12}<0$. Then, except the 
homoclinic loop connecting $p_{1}^{1}$ (resp., $p_{2}$ ), system (1) has no periodic orbits as $\mu \in L_{1 \lambda}^{2}$ (resp., $\mu \in L_{2 \lambda}^{1}$ ).

Remark 8. It is easy to see that homoclinic loop connecting $p_{1}^{0}$ and heteroclinic loop joining $p_{1}^{0}, p_{2}$ cannot be bifurcated from $\Gamma$, which is exactly determined by the generic condition $\left(H_{1}\right)$.

Finally, we consider the case $-\beta \leq x_{0}^{1}<\lambda_{p}$. Due to Figure 1 and (25), it follows from (31) that

$$
\begin{gathered}
s_{2}=\left[\delta^{-1}\left(M_{1 \lambda}^{1} \lambda+M_{1 \mu}^{1} \mu\right)\right]^{\rho_{2}^{1} / \lambda_{2}^{1}}+\text { h.o.t., } \\
x_{0}^{1}=\left(w_{2}^{12}\right)^{-1} \delta s_{2}^{\rho_{2}^{2} / \rho_{2}^{1}}+M_{2 \lambda}^{1} \lambda+M_{2 \mu}^{1} \mu+\text { h.o.t. }
\end{gathered}
$$

Theorem 9. Assume the conditions $\left(H_{1}\right)-\left(H_{3}\right)$ are true, $\operatorname{rank}\left(M_{1 \lambda}^{1}, M_{1 \mu}^{1}\right)>0$ and $\operatorname{rank}\left(M_{2 \lambda}^{1}, M_{2 \mu}^{1}\right)>0$. Then,

(i) there exists a surface

$$
\begin{aligned}
\Sigma_{1}(\mu, \lambda)=\{\mu(\lambda): & {\left[\delta^{-1}\left(M_{1 \lambda}^{1} \lambda+M_{1 \mu}^{1} \mu\right)\right]^{\rho_{2}^{1} / \lambda_{2}^{1}}+\text { h.o.t. }=0, } \\
& -\beta \leq M_{2 \lambda}^{1} \lambda+M_{2 \mu}^{1} \mu+\text { h.o.t. }<\lambda_{p}, \\
& 0<|\mu|, \lambda \ll 1\},
\end{aligned}
$$

such that system (1) has two orbits heteroclinic to $p_{1}^{1}$, $p_{2}, p_{1}^{0}$ as $\mu \in \Sigma_{1}(\mu, \lambda)$;

(ii) there exists a region in the $(\lambda, \mu)$ space

$$
\begin{aligned}
& \Delta=\{(\lambda, \mu):-\beta \leq\left(w_{2}^{12}\right)^{-1} \delta^{\left(\lambda_{2}^{1}-\rho_{2}^{2}\right) / \lambda_{2}^{1}} \\
& \times\left(M_{1 \lambda}^{1} \lambda+M_{1 \mu}^{1} \mu\right)^{\rho_{2}^{2} / \lambda_{2}^{1}} \\
&+M_{2 \lambda}^{1} \lambda+M_{2 \mu}^{1} \mu \\
&+ \text { h.o.t. }<\lambda_{p}, \\
& M_{1 \lambda}^{1} \lambda+M_{1 \mu}^{1} \mu>0, \\
&0<|\mu|, \lambda \ll 1\},
\end{aligned}
$$

such that system (1) has a heteroclinic orbit connecting $p_{1}^{1}$ and $p_{1}^{0}$ for $(\lambda, \mu) \in \Delta$.

Proof. (i) If $s_{2}=0$ in (50), then

$$
\begin{gathered}
0=\left[\delta^{-1}\left(M_{1 \lambda}^{1} \lambda+M_{1 \mu}^{1} \mu\right)\right]^{\rho_{2}^{1} / \lambda_{2}^{1}}+\text { h.o.t., } \\
x_{0}^{1}=M_{2 \lambda}^{1} \lambda+M_{2 \mu}^{1} \mu+\text { h.o.t. }
\end{gathered}
$$

which shows that there exists a surface $\Sigma_{1}(\mu, \lambda)$ such that (50) has a solution $s_{2}=0$ and $-\beta \leq x_{0}^{1}<\lambda_{p}$ for $\mu \in \Sigma_{1}(\mu, \lambda)$, then system (1) has two heteroclinic orbits, one is heteroclinic to $p_{1}^{1}$ and $p_{2}$ and the other is heteroclinic to $p_{2}$ and $p_{1}^{0}$.

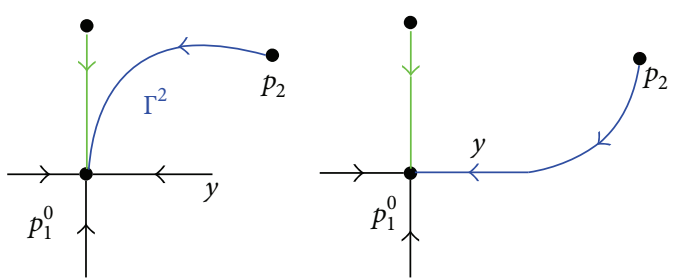

(a)

(b)

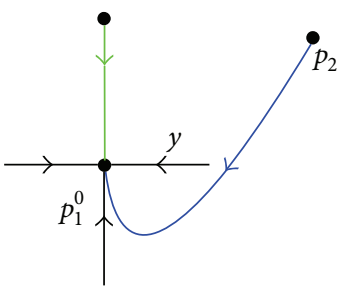

(c)

Figure 2

(ii) If $s_{2}>0$ in (50), one attains $M_{1 \lambda}^{1} \lambda+M_{1 \mu}^{1} \mu>0$. Eliminating $s_{2}$ in (50), we achieve

$$
\begin{aligned}
x_{0}^{1}= & \left(w_{2}^{12}\right)^{-1} \delta^{\left(\lambda_{2}^{1}-\rho_{2}^{2}\right) / \lambda_{2}^{1}}\left(M_{1 \lambda}^{1} \lambda+M_{1 \mu}^{1} \mu\right)^{\rho_{2}^{2} / \lambda_{2}^{1}} \\
& +M_{2 \lambda}^{1} \lambda+M_{2 \mu}^{1} \mu+\text { h.o.t., }
\end{aligned}
$$

which shows that there exists a region $\Delta$ such that when $(\lambda, \mu) \in \Delta$, system (1) has one heteroclinic orbit heteroclinic to $p_{1}^{1}$ and $p_{1}^{0}$.

Remark 10. All the heteroclinic orbits joining $p_{1}^{0}$ will go into $p_{1}^{0}$ in different ways according to different fields of $x_{0}^{1}$; see Figure 2.

\section{Conflict of Interests}

The authors declare that there is no conflict of interests regarding the publication of this paper.

\section{Acknowledgments}

This paper is supported by National Natural Science Foundation of China (nos. 11202192, 11226133), the Fundamental Research Funds for the Central Universities (no. 2652012097), and the Beijing Higher Education Young Elite Teacher Project.

\section{References}

[1] A. J. Homburg and B. Krauskopf, "Resonant homoclinic flip bifurcations," Journal of Dynamics and Differential Equations, vol. 12, no. 4, pp. 807-850, 2000.

[2] C. A. Morales and M. J. Pacifico, "Inclination-flip homoclinic orbits arising from orbit-flip," Nonlinearity, vol. 14, no. 2, pp. 379-393, 2001.

[3] S. Shui, J. Li, and X. Zhang, "Nonresonant bifurcations of heteroclinic loops with one inclination flip," International Journal of Bifurcation and Chaos, vol. 21, no. 1, pp. 255-273, 2011. 
[4] Y. Xu and D. Zhu, "Bifurcations of heterodimensional cycles with one orbit flip and one inclination flip," Nonlinear Dynamics, vol. 60 , no. 1-2, pp. 1-13, 2010.

[5] T. Zhang and D. Zhu, "Codimension 3 homoclinic bifurcation of orbit flip with resonant eigenvalues corresponding to the tangent directions," International Journal of Bifurcation and Chaos in Applied Sciences and Engineering, vol. 14, no. 12, pp. 4161-4175, 2004.

[6] B. Deng, "Homoclinic bifurcations with nonhyperbolic equilibria," SIAM Journal on Mathematical Analysis, vol. 21, pp. 693720, 1990.

[7] J. Klaus and J. Knobloch, "Bifurcation of homoclinic orbits to a saddle-center in reversible systems," International Journal of Bifurcation and Chaos in Applied Sciences and Engineering, vol. 13, no. 9, pp. 2603-2622, 2003.

[8] J. D. M. Rademacher, "Homoclinic orbits near heteroclinic cycles with one equilibrium and one periodic orbit," Journal of Differential Equations, vol. 218, no. 2, pp. 390-443, 2005.

[9] S. Wiggins, Introduction to Applied Nonlinear Dynamical System and Chaos, Spinger, New York, NY, USA, 1990. 


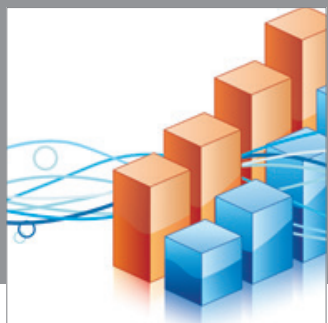

Advances in

Operations Research

mansans

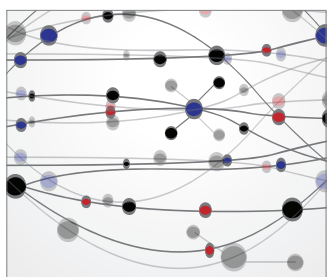

The Scientific World Journal
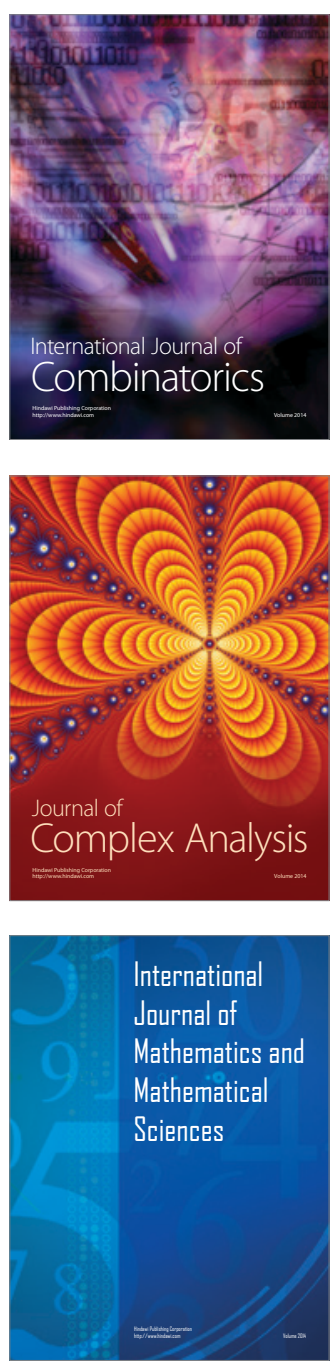
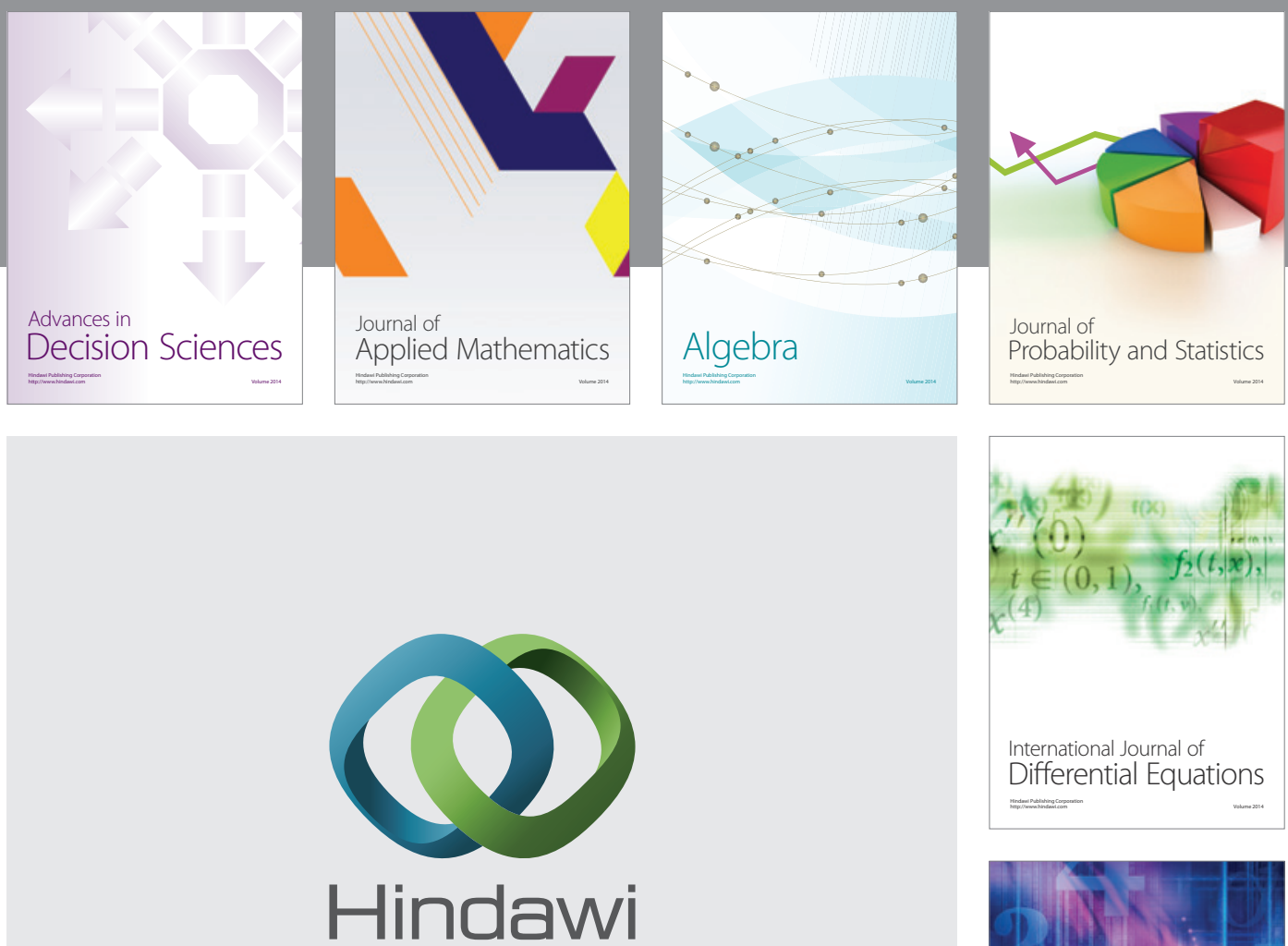

Submit your manuscripts at http://www.hindawi.com
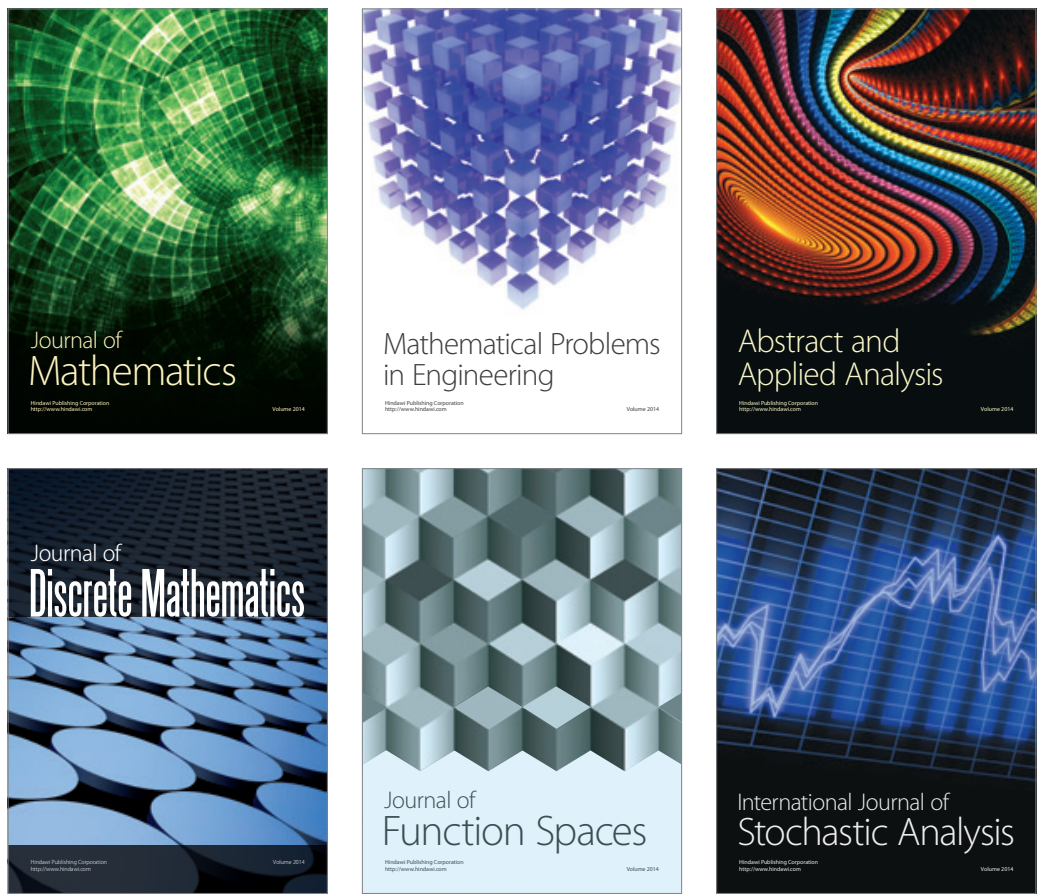

Journal of

Function Spaces

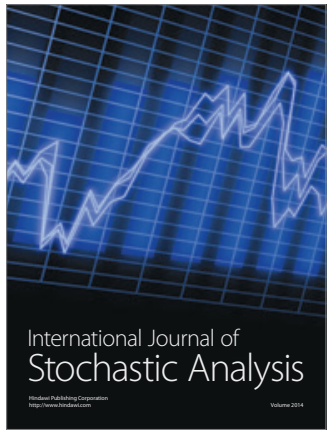

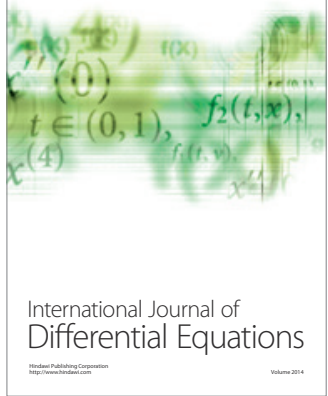
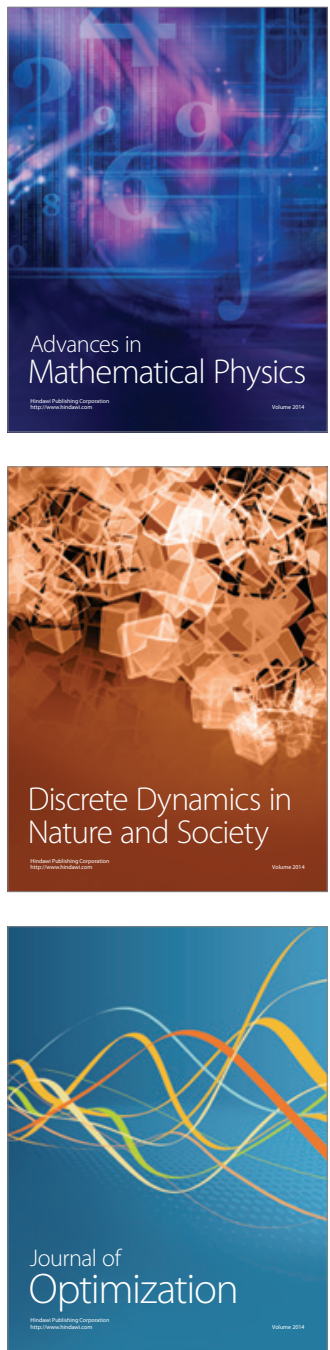